\title{
EL REGLAMENTO CON STITUCIONAL PROVISORIO DE 1812: REFLEXIONES PARA UN BICENTENARIO
}

\author{
THE PROVISIONAL CONSTITUTIONAL STATUTE OF 1812: REFLECTIONS FOR A \\ BICENTENNIAL
}

\section{Cristóbal García-Huidobro Becerra*}

\section{A MODO DEINTRODUCCIÓN}

El 26 de octubre de 2012 se conmemorarán doscientos años de la promulgación del Reglamento Constitucional Provisorio de 1812, que junto con los reglamentos de 1811 y 1814 representan los primeros intentos de entregar a Chile un ordenamiento jurídico que consagrase los primeros pasos de nuestro país hacia la autonomía. Pero sería el reglamento de 1812 el que al canzaría mayor perfección, avanzando de manera más osada en la separación política de Chile con su M etrópoli.

Lamentablemente, ni la historiografía política ni la historiografía del Derecho se ha detenido lo suficiente en el estudio de estos cuerpos jurídicos, que si bien son de corta extensión, imperfectos, y como bien lo señalan sus autores, provisorios, dan el pulso de la política del momento, entregándonos también una perspectiva interesante de cómo en un ámbito empapado de las ideas de las revoluciones atlánticas (norteamericana y francesa), pervivía de una u otra forma la tradición indiana.

El presente ensayo tiene como objeto reflexionar y revisitar algunos de los tópicos más importantes relativos a la creación y promulgación del Reglamento Constitucional Provisorio de 1812, a la vez que busca dar una interpretación sucinta de su contenido, influencias y de la importancia de este mismo para

\footnotetext{
* Profesor de Historia del Derecho, U niversidad AIberto $\mathrm{H}$ urtado, de $\mathrm{H}$ istoria Constitucional de $\mathrm{Chile}$, Universidad Finis Terrae y de $\mathrm{H}$ istoria del Chile en el Siglo XX, Universidad del Desarrollo. Correo electrónico: cagarcib@uc.cl Coordinador General de la Revista Chilena de D erecho. D.Phil (c) in H istory, University of $\mathrm{O}$ xford.
}

encaminar a nuestro país por las vías de la emancipación.

\section{PRIMEROS PASOS HACIA LA AUTONOMÍA}

El 18 de septiembre de 1810, Chile comenzó tímidamente a dirigir sus pasos hacia la autonomía y luego a la independencia por medio de la creación de su primer gobierno nacional. Este, dirigido por la Junta de Gobierno constituida en el cabildo abierto reunido en el Tribunal del Consulado, a poco andar se vio en la necesidad de contar con mayores facultades para el manejo de los asuntos domésticos del país, pero también de un organismo que tuviese a su cargo la creación de las leyes, siguiendo de esta manera la doctrina clásica de separación de funciones administrativas. De tal forma, la Junta de Gobierno decidió convocar a elecciones para formar un Congreso Nacional, que estaría constituido por diputados representantes de todas las provincias del país.

Las elecciones se verificaron el 6 de mayo de 1811, no sin revuelo ni problemas, especialmente por las opiniones que los sectores más exaltados del espectro político de la época tenían sobre dicha institución. La minoría realista, formada por partidarios de mantener el statu quo colonial, vio en la creación de este cuerpo legislativo un intento de separación de la Corona española, e incluso un paso previo para la independencia ${ }^{1}$. Intentaron oponerse a su reunión por medio de la fuerza, apoyando

1 Encina, Francisco Antonio (1970) Historia de Chile, tomo VI (Santiago, Editorial N ascimento) pp. 253-276. 
un motín dirigido por el coronel español Tomás de Figueroa, quien en abril de 1811 trató de derrocar al Gobierno de la Junta de 1810, impidiendo que se llevaran a cabo las elecciones para el Congreso Nacional. Sin embargo, el levantamiento fue rápidamente sofocado por las tropas al servicio de la Junta, y Figueroa fue fusilado bajo el cargo de alta traición?

Por otra parte, los "patriotas", comprometidos con la causa de independizar el país, también desconfiaban de la reunión de un cuerpo cuya formación parecía solo consolidar el poder del mayoritario sector de los "moderados", quienes simplemente pretendían reformar el sistema de gobierno, sin producir un quiebre definitivo con España. De esta manera, los patriotas consideraban que la creación de esta asamblea solo demoraría un proceso que debía ser determinante por su rapidez, y que por sus hechos consumados no permitiese una vuelta al régimen anterior ${ }^{3}$.

No obstante, superando las desconfianzas de un primer momento, el Primer Congreso $\mathrm{N}$ acional de Chile inició sus sesiones el 4 de julio de 1811. "A las diez de la mañana, y al son de una salva de artillería, salía del Palacio la comitiva compuesta por los diputados del Congreso, de los cinco vocales de la Primera Junta de Gobierno, del Cabildo, del nuevo Tribunal de Justicia, y de algunos militares, doctores de la Universidad y vecinos del alto rango, especialmente invitados (... )"4. De este modo inició sus trabajos la primera asamblea legislativa del país.

A poco andar, se determinó que era necesaria la creación de una entidad que detentase el Poder Ejecutivo en forma autónoma. Así, entre el 29 de julio y el 9 de agosto de 1811, se discutió en el seno del Congreso la creación de una Junta Ejecutiva con el nombre de Autoridad Ejecutiva Provisoria, y del reglamento que

\footnotetext{
2 Fray Martínez, M elchor (1964): M emoria histórica sobre la revolución de Chile, tomo I (Santiago, Ediciones de la Biblioteca $\mathrm{N}$ acional).

${ }^{3}$ EnCina (1970) pp. 198 y 251-253.

${ }^{4}$ Barros Arana, Diego (2002) Historia general de Chile, tomo VIII, (Santiago, Editorial U niversitaria).
}

la regiría5. Luego de un sucinto aunque acalorado debate, se dispuso por el Congreso que la Autoridad Ejecutiva quedaría formada por tres miembros, los cuales se alternarían cada mes para desempeñar su presidencia ${ }^{6}$. Constituido entonces, el nuevo Poder Ejecutivo de Chile asumió formalmente sus funciones, detentando el ejercicio de las labores administrativas y de gobierno del país entre el 11 de agosto y el 4 de septiembre de $1811^{7}$.

La mayoría moderada del Congreso $\mathrm{Na}$ cional nombró en los cargos de la nueva Junta a M artín Calvo Encalada, Juan José Aldunate y Francisco Javier del Solar y como su secretario fue designado Judas Tadeo Reyes, a quien se encargó oficialmente las funciones ministeriales de secretario de Gobierno, con preferencia en materias referidas a la mantención del orden y seguridad interior del Reino, constituyéndose así en el primer antecedente político-administrativo del M inisterio del Interior.

Sin embargo, pese a la separación de funciones establecida por el reglamento constitucional redactado por el Congreso $\mathrm{N}$ acional y que daba creación a la Autoridad Ejecutiva, el ejercicio del gobierno interior del país pertenecía, en los hechos, casi enteramente al Congreso ${ }^{8}$. Este último se reservaba la facultad de hacer cumplir las leyes, el ejercicio del patronato eclesiástico, el manejo de las relaciones exteriores, el mando de las armas y la supervi-

${ }^{5}$ Véase Reglamento para el arreglo de la Autoridad Ejecutiva Provisoria de Chile, 1811.

${ }^{6}$ Barros Arana (2002) p. 263. El 14 de agosto se redactó el reglamento de la Autoridad Ejecutiva, en el que se expresaba el deseo de aplicar la separación de poderes y fijar los límites de cada uno. El reglamento estaba compuesto por 13 artículos, en el que se confunden los Poderes Legislativo y Ejecutivo, entregando una preponderancia absoluta al Congreso $\mathrm{N}$ acional. Por ello, la Junta funcionó más como cuerpo dependiente del Congreso que en forma autónoma, como originalmente se había pensando.

7 Sesiones de los Cuerpos Legislativos, tomo I, 1811, Bando del nombramiento de la Segunda Junta de Gobierno por el Congreso $\mathrm{N}$ acional, en 10 de agosto de 1811.

${ }^{8}$ Heise, Julio (1959)H istoria Constitucional de Chile, (Santiago, Editorial Jurídica de Chile) p. 33. 
gilancia de todos los actos del Ejecutivo ${ }^{9}$. De esta manera, la Autoridad Ejecutiva quedaba reducida a una mera creación burocrática encargada de la simple tramitación de negocios administrativos, radicando su único poder en la facultad de control y resguardo de la seguridad que se llevaba a cabo por medio de la Secretaría de la Junta ${ }^{10}$.

En palabras de Ricardo Donoso, este primer intento de organización política y sus frutos, ofrecía "(...) más interés como expresión del estado de la cultura que como tentativa de organización de los poderes públicos, pues es harto revelador de cuan incipientes eran los conocimientos (... ) y cuan vacilantes los primeros pasos que daba la nacionalidad en el cambio de su organización política"11.

$M$ ientras se desarrollaban estos hechos, llegaba al país procedente de España un joven militar que se había batido valientemente contra las tropas napoleónicas que invadían la Península. José Miguel Carrera, perteneciente a una de las familias más ilustres y comprometidas con la causa patriota ${ }^{12}$, pasó rápidamente a convertirse en uno de los líderes políticos más relevantes del período conocido como "Patria Vieja", y fue este quien dio los pasos más osados hacia la creación de un gobierno independiente en una época en que la vacilación se convertía en la nota común de la vida política13.

\section{EL REGLAMENTO CONSTITUCIONAL DE 1812}

Durante los meses subsiguientes a la apertura de las sesiones del Primer Congreso $\mathrm{N}$ acional, la situación política nacional fue enrareciéndose en forma acelerada. Las tensiones entre los distintos grupos políticos fueron deteriorando el frágil equilibrio existente al interior

\footnotetext{
${ }^{9}$ Barros Arana (2002) p. 271.

10 Barros Arana (2002) p. 271.

11 Donoso, Ricardo (1946) Las ideas políticas en Chile, (M éxico D.F., Fondo de Cultura Económica, p. 40.

12 Heise (1959) p. 34.

13 Collier, Simon (1978): I deas y política en la Independencia chilena 1808-1833, (Santiago, Editorial Andrés Bello) pp. 50 y ss.
}

del país. Para empeorar las cosas, el 5 de septiembre de 1811, pocos días después de inauguradas las funciones de la Autoridad Ejecutiva, los diputados representantes de Concepción se marginaron de las sesiones del Congreso, denunciando que la distribución de los escaños del cuerpo legislativo favorecía ampliamente a Santiago ${ }^{14}$.

Posteriormente, entre septiembre y noviembre de 1811, se sucedieron en Santiago dos golpes de Estado que cambiaron la fisonomía del proceso político en Chile. El golpe del 4 de septiembre de 1811, dirigido por José M iguel Carrera, forzó la creación de una nueva Junta de Gobierno, compuesta esta vez por Juan Enrique Rosales, Juan M artínez de Rozas, M artín Calvo Encalada, Juan M ackenna y Gaspar M arín. Asimismo, el Congreso debió consentir en el alejamiento de ocho de sus miembros y en la inclusión de otros más proclives a la tendencia patriota $y$, en algunos casos, abiertamente independentista. Sin embargo, al mismo tiempo que esto ocurría en Santiago, en Concepción estalló una revuelta regionalista, dirigida por el mismo Rozas, quien pese a su nombramiento en la nueva Junta de Santiago, buscaba hacerse con el poder, convirtiendo a Concepción en una provincia autónoma. Como resultado, el 5 de septiembre de 1811 se formó una Junta de Gobierno en dicha ciudad, detentando toda la autoridad, facultades y privilegios de gobierno y administración de la Provincia, y aunque se declaraba dependiente de la autoridad de la capital, para todos los efectos prácticos la provincia actuaba en forma independiente del resto del país, convirtiéndose en una entidad política autónoma ${ }^{15}$.

14 Barros Arana (2002) pp. 268-270.

15 Para los antecedentes de este movimiento, puede consultarse Cartes, Armando (2010): Concepción contra Chile (Santiago, Ediciones $\mathrm{C}$ entro de Estudios Bicentenario, passim. Cabe señalar que al momento en que estalló el golpe de Estado en Santiago, al día siguiente Concepción creó su propia Junta. Evidentemente las noticias de los acontecimientos de Santiago no pudieron llegar tan rápido a Concepción, y si se toma en cuenta que Rozas y Carrera formaban un tandem ideológico para ese entonces, es posible argüir que ambos actuaron de común acuerdo. Sin embargo, al pasar el tiempo, 
El 15 de noviembre de 1811, un segundo golpe de Estado, dirigido esta vez por Juan José C arrera, terminó por elevar a su hermano José M iguel a la presidencia de una nueva Junta de Gobierno. Esto y el cierre definitivo de las sesiones del Primer Congreso $\mathrm{N}$ acional el 2 de diciembre del mismo año consolidaron el poder de Carrera en la capital ${ }^{16}$. N o obstante lo anterior, el golpe en Santiago no hizo otra cosa que alejar a las provincias de Concepción y Santiago aún más, ya que la amenaza de la instauración de un gobierno despótico en el país había fortalecido la posición de Rozas, quien seguía gobernando sin mayor contrapeso en el sur del país.

No obstante lo anterior, durante la primera parte de 1812 los gobiernos de Santiago y Concepción intentaron llegar a un acuerdo sobre cómo organizar la causa patriota y darle al país un gobierno único, ya que se sabía que los españoles estaban preparando una expedición contra Chile desde el Virreinato del Perú, por lo que era necesario aunar voluntades frente a la potencial amenaza que se cernía sobre el país. Si bien en un primer momento hubo acuerdo entre ambas provincias, Santiago terminó por desconocer los términos de su arreglo con Concepción e inició una movilización militar que llevó al país a una breve guerra entre ambas ciudades, terminando con la derrota de las fuerzas penquistas y la consolidación de Carrera como líder indiscutido del país ${ }^{17}$. Este impulsó enérgicamente el sentido revolucionario de las reformas que se implementaban desde 1810. Sin embargo, gran parte de la elite, tanto en Santiago como en las ciudades del sur, veían con poco entusiasmo y más bien con desconfianza las pretensiones del joven caudillo. Así entonces, la ciudadanía exigió de Carrera una constitución sancionada por el pueblo soberano, para poner

la relación entre los dos próceres degeneró en una fuerte rivalidad. Finalmente Rozas fue derrocado en Concepción y esta se rindió finalmente a Santiago a mediados de 1812 .

16 Heise (1959) pp. 34-35 (1946) Barros Arana, pp. 339-359 y D onoso, pp. 40-41.

17 Archivo N acional-Fondo Sergio Fernández Larraín, vol. 32, pieza 23, Tratados y proposiciones entre las provincias de Santiago y Concepción. Enero-M ayo de 1812. fin a cualquier intento de instaurar en el país el despotismo y la arbitrariedad ${ }^{18}$.

Una comisión compuesta por Francisco Antonio Pérez, Camilo Henríquez, Manuel de Salas, Antonio José de Irrisarri, Francisco de la Lastra, H ipólito de Villegas y Jaime Zudáñez fue elegida para redactar un reglamento constitucional de carácter provisional, mientras se estabilizaba la situación del país y se daban las condiciones para convocar a un Congreso con el fin de que redactara una constitución definitiva.

Para agilizar la labor de la comisión, esta trabajó habiendo utilizado como base un preproyecto con reminiscencias federalistas redactado por el cónsul de los Estados Unidos, Joel Robert Poinsett ${ }^{19}$, y otro de Agustín Vial, quien obraba como Secretario de Gobierno de la Junta de Gobierno ${ }^{20}$.

El proyecto constitucional fue promulgado por C arrera el 26 de octubre de 1812, y a modo general estableció un sistema de división clásica de los poderes del Estado. Un Ejecutivo colegiado, con el nombre de "Junta de Gobierno", tendría a su cargo la administración del país - con amplios poderes, incluyendo por ejemplo la facultad de poder arrestar personas por crímenes contra el Estado-; el Poder Le gislativo recaería en un Senado, encargado de redactar las leyes, mientras que el Poder Judicial sería detentado por los tribunales de justicia21.

Aunque su redacción era imperfecta, acotada y carecía de la tecnicidad jurídica que se esperaría de un documento que sienta las bases del obrar del Estado, el Reglamento de 1812

18 Silva Castro, Raúl, (1959): Egaña en la Patria Vieja 1810-1814 (Santiago, Editorial Andrés Bello) pp. 75-81 y Galdames, Luis (1925): La Historia de Chile. La evolución constitucional (Santiago, Imprenta Universitaria) p. 321.

19 Lamentablemente no se tiene registro de dicho documento, salvo que daba a Chile el nombre de "Provincias Unidas de Chile". Luis G aldames (1925) p. 322, pone en duda la existencia dicho documento. 20 M iguel Varas Velásquez (1915): El reglamento constitucional de 1812. Nuevos documentos", en Revista Chilena de Historia y Geografía $\mathrm{N} 018$, pp. 108-109.

${ }^{21}$ Cumming, Alberto (1913): El reglamento Constitucional de 1812 (Santiago, Imprenta Universitaria) pp. 12-13. 
se constituyó en una de las más claras muestras de ejercicio de la naciente soberanía nacional. Ya por medio de su artículo $\mathrm{V}$, si bien se reconocía a Fernando VII como Rey y soberano señor de Chile, prohibía en forma expresa que se obedecieran órdenes o se siguieran instrucciones emanadas de cualquier autoridad que no se encontrase radicada dentro de las fronteras del país, poniéndose en franca rebelión contra el poder colonial españo| ${ }^{22}$.

Sin embargo, cabe señalar que si bien el entusiasmo provocado por la promulgación del Reglamento fue considerable, ni en Concepción ni en Coquimbo - las otras dos provincias del Reino- se vieron con buenos ojos los eventos de Santiago. Como había sido durante la época colonial, la capital de Chile amenazaba con afianzarse nuevamente como el centro en la toma de decisiones y de la administración. Ya en 1811 Concepción había hecho sentir su disconformidad con la composición del Congreso $\mathrm{N}$ acional, para luego erigir un gobierno propio que posteriormente desencadenaría una "guerra" entre la capital del sur y Santiago. Por su parte Coquimbo manifestó por medio de un cabildo abierto efectuado en La Serena que no juraría el reglamento hasta que la provincia no deliberase sobre su articulado, e incluso, siguiendo los pasos de Concepción, formó su propia Junta de G obierno ${ }^{23}$.

Así las cosas, la promulgación del Reglamento de 1812 no fue cosa pacífica. Las tensiones interprovinciales producto del centralismo santiaguino, sumado a la amenaza creciente de que Carrera, apoyado por su adeptos, se convirtiera en un déspota, enturbiaba y enrarecía los fundamentos en los que se inspiró el Reglamento: libertad de imprenta, igualdad de derechos, seguridad personal, protección de la propiedad $^{24}$, y en definitiva todos los principios liberal-revolucionarios de las revoluciones atlánticas.

$22 \mathrm{Cfr}$. Valencia, en cuanto al texto del Reglamento Constitucional de 1812. Véase también Heise (1959) pp. 34-35.

${ }^{23}$ Archivo N acional-Fondo José I gnacio Víctor Eyzaguirre, vol. 20, Comunicación de la Junta de Santiago a la de Coquimbo, 9 de marzo de 1814.

${ }^{24}$ Varas (1915) p. 116.

\section{CAMBIO Y CONTINUIDAD}

Autores del auge historiográfico de la segunda mitad del siglo XIX como Domingo Santa M aría, Melchor Concha y Toro y Federico Errázuriz Zañartu ${ }^{25}$ concuerdan en que la inspiración principal del movimiento constitucionalista en Chile provino de Francia y los Estados Unidos; afirmación que se repite en estudios posteriores hechos por Diego Barros Arana ${ }^{26}$, José Victorino Lastarria ${ }^{27}$, M iguel Luis Amunátegui28, Ricardo Donoso ${ }^{29}$, Domingo Amunátegui Solar ${ }^{30}$ y varios otros. Incluso desde la perspectiva de la Historia del Derecho, autores como Jorge Huneeus ${ }^{31}$, Jaime Eyzaguirre ${ }^{32}$ y $\mathrm{M}$ ario Verdugo recalcan el valor revolucionario de nuestros textos constitucionales ${ }^{33}$. Para el caso del Reglamento Constitucional Provisorio, el asunto no

25 Cfr. Santa María, Domingo (1858): M emoria histórica sobre los sucesos ocurridos desde la caída de D. Bernardo O'H iggins en 1823 hasta la promulgación de la Constitución dictada en el mismo año (Santiago, Imprenta del País); Melchor Concha y Toro (1862) Chile entre 1824 y 1828 (Santiago, Imprenta Chilena) y Errázuriz, Federico (1861) Chile bajo el imperio de la Constitución de 1828 (Santiago, Imprenta Chilena).

${ }^{26}$ Barros Arana (2002) .

27 Lastarria, José Victorino (1853): Historia cons titucional de medio siglo (Valparaíso, Imprenta del Mercurio).

28 Amunátegui, M iguel Luis (1911): La crónica de 1810, 3 vols. (Santiago, Imprenta y Litografía Barcelona).

29 D onoso (1946) .

30 Amunátegui Solar, D omingo (1930): N acimiento de la República de Chile 1808-1833 (Santiago, Establecimientos G ráficos Balcells).

31 Huneeus, Jorge (1879-1880): La Constitución ante el Congreso, 2 vols. (Santiago, Imprenta Los Tiempos).

32 Eyzaguirre, Jaime (2010): I deario y ruta de la emancipación chilena (Santiago, Editorial Universitaria) y del mismo autor (1997): H istoria de las instituciones políticas y sociales en Chile (Santiago, Editorial Universitaria).

33 Verdugo, M ario (1930): Los principios del constituci onalismo clásico en los ordenamientos fundamentales de C hile en Revista de D erecho Público N 19-20, pp. 117-143. 
cambia mucho. De su lectura es clara la influencia revolucionaria, siendo ya el mismo término "constitucional" un reflejo de su sesgo antiabsolutista y republicano, lo que implica un alejamiento de la tradición jurídica indiana. Sin embargo, luego de tres siglos de existencia y uso, esta no podía desaparecer por decreto y siguió permeando los primeros textos constitucional es chilenos, incluso ya bien desarrollada la etapa republicana de nuestra historia ${ }^{34}$.

\subsection{Los principios de la Revolución}

La separación de funciones administrativas, propias de la literatura política ilustrada, estaba consagrada en los artículos III, VII y XVII que regulaban las funciones Ejecutiva, Legislativa y Judicial, respectivamente ${ }^{35}$. Asimismo, se establecía la el ectividad de los cargos, ya sea del Ejecutivo o del Legislativo, e incluso de los gobiernos municipales representados en los Cabildos, estipulado esto último en el artículo XII del Reglamento de 1812.

Pero existían también otros principios fundamentales del Reglamento que eran propios del pensamiento revolucionario e ilustrado: el aseguramiento de una serie de libertades como la de imprenta (Art. XXIII) y la libertad ambulatoria (Art. XXIV), así como también se consagró una protección preferente sobre el derecho de propiedad (Art. XVI), dándose además los primeros pasos para establecer un medio de publicidad respecto de las finanzas públicas que asegurase la responsabilidad de las autoridades con relación a su manejo (Art. XXV).

En el orden administrativo doméstico, quizás la innovación más relevante del Reglamento Constitucional estaba contenida en su artículo XIV, que creaba dos secretarías, una encargada de los negocios domésticos y de la seguridad interior con el nombre de "Secretaría de Gobierno", y otra encargada de las relaciones exteriores del Reino, con el nombre de

\footnotetext{
34 Bravo Lira, Bernardino (1996): El estado de derecho en la historia de Chile (Santiago, Ediciones Universidad Católica de Chile) passim.

35 Varas (1915) pp. 118-124.
}

"Secretaría de Relaciones Exteriores"36. Al día siguiente de la promulgación del reglamento, las secretarías fueron provistas, y por primera vez en la historia del país, Chile tuvo ministerios propios. La cartera de Gobierno quedó formalmente encargada de las funciones de administración doméstica y de mantención del orden y seguridad al interior de las fronteras del país, siendo nombrado como Secretario de Gobierno, Agustín Vial Santelices ${ }^{37}$, quien luego se convertiría en Secretario de Interior al cambiarse el nombre de dicha cartera el 3 de noviembre de 1812.

Cabe mencionar también que el Reglamento Constitucional Provisorio de 1812 dedicaba una parte importante de su articulado a establecer garantías inscritas dentro del ámbito del procedimiento penal, inspiradas por los ideales ilustrados, que incluirían las reflexiones del $M$ arqués de Beccaria en su famoso tratado $D$ e los delitos y las penas ${ }^{38}$. En efecto, uno de los redactores del Reglamento, Fray Camilo $\mathrm{H}$ enríquez, fue un ávido promotor de las idea de Beccaria en Chile, y pareciera que su influencia se trasuntó en la redacción del Reglamento de 1812. A modo de ejemplo, el artículo XXI del Reglamento establece que "Las prisiones serán lugares cómodos y seguros para la detención de las personas (... y y de ningún modo servirán

36 El texto literal del artículo en cuestión señalaba expresamente lo siguiente: "XIV.- Para el despacho de los negocios habrán dos Secretarios, el uno para los negocios del reino, y el otro para las correspondencias de fuera".

37 Barros Arana (2002) pp. 426-434. Véase también, en la parte correspondiente a la composición de los ministerios, el trabajo de Luis Valencia ya antes citado.

${ }^{38}$ Donoso, Sebastián (1997): Fuentes de los derechos y garantías individuales en el Reglamento Constitucional Provisorio de 1812 (Santiago, Tesis para optar al grado de Licenciado en Derecho por la Pontificia Universidad Católica de Chile. Cabe señalar que el contenido de esta investigación se encuentra publicado en formato digital por el Instituto de Investigaciones $\mathrm{H}$ istóricas J osé M iguel Carrera, pero lamentablemente no se especifican mayores datos respecto de qué año data la publicación. Es por este motivo que se ha preferido acudir a la fuente principal, cual es la tesis de grado antes citada. 
para mortificar delincuentes". El espíritu redentor y filantrópico de la Ilustración se presenta en forma clara, y el mismo $\mathrm{H}$ enríquez señaló en una ocasión que las "venerables máximas" de Beccaria han recibido la sanción augusta de las leyes ${ }^{39}$. Asimismo, el mismo Reglamento es profuso en garantizar la existencia de un debido proceso judicial, con penas proporcionales al delito que se busca castigar (Art. XVIII), asegurando la protección del reo y su acceso debido al juez natural llamado por la ley a conocer del caso particular ${ }^{40}$.

\subsection{Autonomía y Soberanía}

Si bien desde el punto de vista de los derechos fundamentales, el Reglamento Constitucional Provisorio de 1812 pareciera haber sentado las bases de lo que serían los futuros catálogos de derechos inclusos en nuestras $\mathrm{C}$ artas Fundamentales, para el momento específico de su promulgación, estas materias no fueron las más radicales ni tampoco las más relevantes. Fueron las declaraciones de soberanía y autogobierno establecidas en su articulado las que convirtieron a esta pieza de legislación fundamental en una verdadera declaración de autonomía nacional.

Tanto el reglamento de la Junta de 1810 y el Reglamento Provisional de 1811 poseían un lenguaje moderado y hasta tímido al momento de referirse a los principios del autogobierno, pero el Reglamento de 1812 no escatima palabras para dejar en claro sus objetivo de servir como pivote para la redacción de una constitución permanente. Ya en su preámbulo se hacía referencia a la necesidad de que Chile se autogobernase por autoridades el egidas de entre sus "ciudadanos" a través del sufragio, rechazando al gobierno provisional constituido en España hasta el retorno de Fernando VII.

Por otra parte, y actuando de forma soberana, el mismo Reglamento de 1812 establece en su artículo II que "El pueblo hará su Consti-

39 Amunátegui Aldunate, Miguel Luis (1889): Camilo Henríquez, tomo I (Santiago, Imprenta $\mathrm{Na}$ cional) p. 111.

40 Donoso (1997) V éanse las referencias respecto a los artículos XIX al XXII del Reglamento Constitucional Provisorio. tución por medio de sus representantes", y acto seguido, si bien reconoce a Fernando VII como Rey, le fuerza a aceptar una futura C onstitución y establece un Ejecutivo nacional, prescindiendo de las autoridades peninsulares. Pero la más directa de todas las declaraciones soberanas contenidas en el Reglamento se encuentra en su artículo V, cuyo tenor señalaba que "N ingún decreto, providencia u orden, que emane de cualquiera autoridad o tribunales de fuera del territorio de Chile, tendrá efecto alguno; y los que intentaren darles valor, serán castigados como reos de Estado".

El artículo en cuestión radicaba las potestades legislativas en Chile, algo que bajo el régimen borbónico estaba estrictamente limitado a la Metrópoli. A mayor abundamiento, en lo relativo a materias jurisdiccionales, las sentencias judiciales emanadas de tribunales extranjeros no tendrían valor alguno en el país, por lo que a través del artículo $\mathrm{V}$ del Reglamento Constitucional de 1812 se cortaban prácticamente todos los lazos que mantenían a la Capitanía General de Chile unida al Reino de España, salvo por el reconocimiento nominal de Fernando VII como monarca. N o es de extrañar entonces, que luego de estos hechos, y viendo la radicalización del movimiento autonomista ${ }^{41}$, el Virrey del Perú, Fernando de Abascal, decidiera poner fin prontamente al experimento chileno enviando tropas, primero dirigidas por el Almirante Antonio Pareja, seguido por el Brigadier $\mathrm{G}$ abino $\mathrm{G}$ aínza y final mente por $\mathrm{M} \mathrm{a}$ riano 0 sorio, para que pusieran en regla a los rebeldes de $\mathrm{C}$ hile.

41 Influenciado también por los avances de los partidarios de la Independencia en Venezuela, Nueva Granada y las Provincias Unidas del Río de la Plata. Véanse Miron Burgin (1987): Aspectos económicos del federalismo argentino (Buenos Aires, Ediciones Solar); Bushnell, D avid (2011): The Santander Regime in Gran Colombia (London, Nabu Press); Earle, Rebeca (ed.) (2002); Rumours of Wars: Civil Conflict in Nineteenth-Century Latin America (London, U niversity of London) y GaravagLia, Juan Carlos (2007) Construir el estado, inventar la nación. El Río de la Plata siglos XVIII-XIX (Buenos Aires, Prometeo Libros). 


\subsection{LA HERENCIA INDIANA}

La historiografía chilena del siglo XIX se empeñó fuertemente en deslindar cualquier herencia española en la temprana creación del ordenamiento jurídico y constitucional chileno. Esto no es de extrañar, ya que, por un lado, el surgimiento de Chile como un país independiente fue fruto de una larga y amarga guerra civil que dividió a la sociedad chilena y sumió al país en los vaivenes de las revoluciones hispanoamericanas. Asimismo, y del caso es decirlo, durante el periodo de la Reconquista o Contrarrevolución de la Independencia42, las autoridades españolas sellaron el recuerdo de su administración en Chile mediante las medidas de represión tomadas durante el periodo 18151817. Finalmente, también es del caso señalar que el rechazo de la tradición indiana o colonial cumplió un efecto pedagógico al momento de instruir a la población sobre el nuevo modelo de gobierno - la República- en contraposición a la M onarquía, enfrentando también los principios revolucionarios de la Independencia a los de la administración española.

Sin embargo, la herencia histórica y jurídica de nuestro pasado colonial no puede borrarse simplemente por decreto. Así entonces, es posible encontrar en nuestros textos legales y constitucionales resabios e influencias de nuestro pasado colonial, no siendo el Reglamento de 1812 una excepción, manteniendo el paradigma de que en todo cambio que se produce, se mantienen elementos de continuidad con el pasado.

Ya en el Preámbulo del Reglamento de 1812 se hace mención a los motivos que han llevado a los chilenos a generar su propio gobierno y un marco constitucional en el que este se desempeñe. Pero importante también es la consideración que se hace respecto como los españoles han solventado la acefalía de su propio gobierno luego del secuestro de Fernando VII. Faltando el titular de la Corona, el poder se retrotrajo al pueblo español, y de la misma

42 Tomo el término de Guerrero Lira, Cristián (2002): La contrarrevolución de la Independencia de Chile (Santiago, Editorial U niversitaria-CID BA). forma "con igual derecho y necesidad mayor"43, el pueblo chileno imitó la conducta de España. D e este modo, se reconoce la doctrina tradicional sobre el origen del poder, que encuentra su fuente en la reversión de los poderes al pueblo a falta del Soberano, propio de la escolástica. Chile conservó su carácter de monarquía bajo la égida de Fernando VII como su Soberano. Asimismo, la tradición de gobierno provincial y comunal por medio de los cabildos también permaneció, manteniendo la tradición electiva de cada lugar para la generación de sus miembros, tal como lo determinaba la Recopilación de Leyes de Indias ${ }^{44}$.

Desde el punto de vista religioso, también existe continuidad entre la legislación indiana y la revolucionaria. El artículo I del Reglamento de 1812 establece que la religión C atólica A postólica "(...) es y será siempre la de Chile", manteniendo la tradicional protección (y control) que la Corona Española mantenía sobre la I glesia C atólica ${ }^{45}$.

Finalmente cabe destacar la protección preferente que otorga el Reglamento Constitucional Provisorio a ciertos derechos, que ya se encontraban reconocidos con anterioridad por la legislación indiana, tales como la seguridad personal, la inviolabilidad del hogar y de los papeles privados ${ }^{46}$, los principios que informan el debido proceso y el resguardo de la integridad personal de los reos encarcelados. Todas estas medidas encontraban su reflejo en normas contenidas en las Siete Partidas, las O rdenanzas

43 Reglamento Constitucional Provisorio de 1812, Preámbulo.

44 Dougnac, Antonio (2000): El sistema jurídico indiano en el constitucionalismo chileno durante la Patria Vieja (1810-1814), en Revista de Estudios H istórico-Jurídicos, N ㅇ XXII, p. 253

45 Del texto impreso del Reglamento de 1812 se suprimió la palabra "romana". Varias explicaciones se han dado sobre el tema, desde que fue a propósito de no reconocer a ninguna otra autoridad salvo las propiamente chilenas, hasta de que se trató de un error de imprenta. Sin embargo, en algunas copias del Reglamento, como la que se le enviara al o bispo de Concepción para su revisión, mantenían la palabra "romana" en su texto. Cfr. Dougnac (2000) p. 250. 46 Véase Art. XVI del Reglamento Constitucional Provisorio de 1812. 
Reales de Castilla y la Recopilación de Leyes de Indias ${ }^{47}$.

\section{A MODO DECONCLUSIÓN}

Al cumplir 200 años del Reglamento Constitucional Provisorio de 1812, también celebramos otro los bicentenarios de ese arco de efemérides que recuerdan los hechos acontecidos entre 1810 y 1818. El año 2010 estuvo marcado por la conmemoración de los 200 años de nuestro primer gobierno nacional. Durante el año 2011, aunque con menos bombos y platillos, se celebraron los 200 años de nuestro Poder Legislativo. En este 2012 también cabe mirar hacia atrás y rescatar del polvo y del olvido los primeros intentos por dar a Chile una constitución en forma que sobrepasase lo meramente reglamentario y adquiriera mayor consistencia jurídica, acercándose paulatinamente a la creación de un sistema constitucional nacional.

Al recordar la promulgación del Reglamento de 1812, traemos nuevamente al gran banquete de la memoria histórica, aquel hito fundamental que significó ese documento, que en su texto contenía el germen de los principios políticos de las revoluciones atlánticas, a la vez que se incluían las primeras declaraciones de soberanía y autogobierno, con un lenguaje que en la práctica significaba la separación de C hile del ámbito de control del Imperio Español. Sin embargo, el Reglamento de 1812 también es un recordatorio de nuestro pasado jurídico coIonial, que eventualmente se imbricó y fusionó con los principios de la Independencia y la Soberanía $\mathrm{N}$ acional, y que se desprenden del texto constitucional de 1812.

Es la síntesis entre lo revolucionariopatriota y lo indiano-colonial, lo que ha servido como bloque en la construcción de la identidad jurídica constitucional americana, y para el caso de Chile, el Reglamento de 1812 es uno de esos primeros ladrillos que sirvieron como base para erigir con solidez el edificio que constituye nuestro ordenamiento jurídico.

${ }^{47}$ Dougnac (2000) pp. 255-257. 
